\title{
ON THE PSEUDO-PERIODS OF THE WEIERSTRASS ZETA FUNCTIONS II*
}

\author{
MAURICE HEINS
}

To Professor Kiyoshi Noshiro on the occasion of his sixtieth birthday.

1. In a recent note bearing the same title (SIAM Journ. of Numerical Analysis, Vol. 3, 1966), I showed that there existed Weierstrass zeta functions having a vanishing pseudo-period. Cf. line 8, p. 376 of Saks-Zygmund, Analytic Functions. Professor Peter Henrici kindly pointed out to me that the mere existence of a Weierstrass zeta function with a vanishing pseudo-period was readily concluded from a classical formula of the theory of elliptic functions (Hurwitz-Courant, Funktionentheorie, 5th ed., p. 210) which reduces the question to the study of the zeros of

$$
\frac{1}{12}-\sum_{1}^{\infty} \frac{k x^{k}}{1-x^{k}},|x|<1
$$

Here the presence of a zero on $] 0,1[$ is obvious. However a much stronger result was implicit in my note since the argument there employed serves to show that any ordered pair of complex numbers, not both zero, appears as the pseudoperiod pair of some Weierstrass zeta function, and in fact even more.

The argument of the cited note is based on the study of a system of functional equations associated with the modular group, the use of the modular function $\lambda$, and the big Picard theorem. Thus it may be said that the note treated a theme of the classical Weierstrass function theory with methods that draw in some part on ideas stemming from the Weierstrass theory. Professor Krishna Chandrasekharan remarked to me that the study of systems of functional equations of the kind treated in the cited note were of interest for Fuchsian and Fuchsoid groups in general. Actually, certain systems of this more general type were studied in an earlier paper of mine (A generalization of the AumannCarathéodory "Starrheitssatz", Duke Math. J. 1941).

In the present paper we study the pseudo-period pairs of the Weierstrass

* Work on this paper was carried out during the tenure of National Science Foundation Grant NSFGP 3432 . 
zeta functions. The system of functional equations entering into the treatment of the question will be handled with the aid of part of the theorem of Denjoy and Wolff concerning holomorphic maps of the open unit disk into itself.

2. We start with an ordered pair $\left(\omega_{1}, \omega_{2}\right)$ of complex numbers satisfying $\operatorname{Im}\left(\bar{\omega}_{1} \omega_{2}\right) \neq 0$. We let $\Omega$ denote the additive subgroup of $\widetilde{c}$ generated by $\left\{\omega_{1}, \omega_{2}\right\}$. By the Weierstrass zeta function associated with $\Omega$, which we denote by $\zeta$, is meant the meromorphic function on $\mathbb{c}$ assigning to $z$ the value

$$
\frac{1}{z}+\Sigma\left(\frac{1}{z-\omega}+\frac{1}{\omega}+\frac{z}{\omega^{2}}\right)
$$

the sum being taken over the nonzero members of $\Omega$. For each $\omega \in \Omega$ we have

$$
\zeta(z+\omega)=\zeta(z)+\eta(\omega),
$$

where $\eta(\omega) \in \mathfrak{C}$. We term $\left(\eta\left(\omega_{1}\right), \eta\left(\omega_{2}\right)\right)$ the pseudo-period pair of $\zeta$ associated with $\left(\omega_{1}, \omega_{2}\right)$. Our goal is to show the following theorem:

The map $\left(\omega_{1}, \omega_{2}\right) \rightarrow\left(\eta\left(\omega_{1}\right), \eta\left(\omega_{2}\right)\right)$ maps $\left\{\operatorname{Im}\left(\bar{z}_{1} z_{2}\right) \neq 0\right\}$ onto $\left\{\left|z_{1}\right|+\left|z_{2}\right|>0\right\}$. Further, each point of $\left\{\left|z_{1}\right|+\left|z_{2}\right|>0\right\}$ has infinitely many non-equivalent preimages with respect to this map.

Here it is understood that $\left(\omega_{1}{ }^{\prime}, \omega_{2}{ }^{\prime}\right)$ is equivalent to $\left(\omega_{1}, \omega_{2}\right)$ provided that $\omega_{1}{ }^{\prime}=\sigma\left(a \omega_{1}+b \omega_{2}\right), \omega_{2}{ }^{\prime}=\sigma\left(c \omega_{1}+d \omega_{2}\right)$, where $\sigma \in \mathbb{C}-\{0\}$ and $a, b, c, d$ are real integers satisfying $|a d-b c|=1$.

It is well-known that $\left(\eta\left(\omega_{1}\right), \eta\left(\omega_{2}\right)\right) \neq(0,0)$ for allowed $\left(\omega_{1}, \omega_{2}\right)$. Further, $\eta(\omega)$ is homogeneous of degree -1 with respect to $(\Omega, \omega)$. We consider normalized $\left(\omega_{1}, \omega_{2}\right)$ of the form $(1, \alpha), \operatorname{Im} \alpha \neq 0$,

and introduce

$$
F(\alpha)=\eta(\alpha) / \eta(1)
$$

The function $F$ takes the value $-i$ at $i$, is meromorphic on $\widetilde{E}-R$ and satisfies the system of functional equations

$$
F[S(\alpha)]=S[F(\alpha)]
$$

for every Möbius transformation $S$ belonging to the modular group, that is, for $S$ of the form

$$
S(z)=(a z+b) /(c z+d),
$$

where $a, b, c, d$ are real integers satisfying $a d-b c=1$. The verification of (2.2) is readily made on noting the fact that for given $\left(\omega_{1}, \omega_{2}\right)$ the map $\omega \rightarrow \eta(\omega)$ is a 
homomorphic map of $\Omega$ into $\widetilde{S}$ considered additively and also the homogeneity of degree -1 of $\eta$. Cf. our note of the same title cited above.

We observe that thanks to the definition of $F$ the theorem of this paper will be established once it is shown that $F$ attains any given value of the extended plane at infinitely many points of $5-R$ which are mutually non-equivalent relative to the modular group. The reduced result will appear as a consequence of two lemmas to be proved below.

3. For the first lemma we shall want a part of the theorem of Denjoy and Wolff concerning holomorphic maps of the open unit disk into itself. It may be stated as follows:

Let $f$ be a holomorphic map of the open unit disk into itself which possesses no fixed point. Then there exists a unique $\eta,|\eta|=1$, such that

$$
\operatorname{Re}\left[\frac{\eta+f(z)}{\eta-f(z)}\right] \geq \operatorname{Re}\left(\frac{\eta+z}{\eta-z}\right),|z|<1 .
$$

We include a brief proof for the sake of completeness. The uniqueness of $\eta$ is clear on geometric grounds. For otherwise there would exist $\eta_{1},\left|\eta_{1}\right|=1$, $\eta_{1} \neq \eta$, satisfying the same property as $\eta$. There would then exist oricycles tangent to the unit circumference at $\eta$ and $\eta_{1}$ respectively and tangent to one another at a point of the open unit disk. By (3.1) this point would be a fixed point of $f$. The hypothesis is contradicted. The existence of $\eta$ may be concluded with the aid of Rouche's theorem and the lemma of Schwarz-Pick. Indeed, let $0 \leq r<1$ and introduce $f_{r}(z)=f(r z),|z|<1$. By Rouché's theorem $f_{r}$ has a unique fixed point, say $\beta(r)$. At the very least we have

$$
\lim _{r \rightarrow 1}|\beta(r)|=1 \text {, }
$$

for otherwise $f$ would have a fixed point. Further, by the lemma of SchwarzPick we have

$$
1-\left|\frac{f(r z)-\beta(r)}{1-\overline{\beta(r) f(r z)}}\right|^{2} \geq 1-\left|\frac{z-\beta(r)}{1-\overline{\beta(r) z}}\right|^{2}
$$

or equivalently,

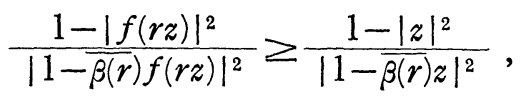

for $0 \leq r<1,|z|<1$. Taking a sequence of $r$ tending to 1 such that the 
corresponding sequence of $\beta(r)$ possesses a limit, say $\eta$, we conclude (3.1) from (3.2).

4. We turn to the first of the two lemmas. Here $\Gamma$ is a group of Möbius transformations mapping the upper half-plane onto itself which is properly discontinuous at each point of $5-R$ but at more than two points of $R \cup\{\infty\}$ fails to be properly discontinuous.

Lемма A. Let $f$ be holomorphic on the upper half-plane and not take any real value. If $f$ satisfies the system of equations

$$
f[S(z)]=S[f(z)],
$$

$\operatorname{Im} z>0, S \in \Gamma$, then $f$ is the identity map.

Proof. We consider first the case where $f$ maps the upper half-plane into itself. Suppose that $f$ is not the identity. If $f$ has a fixed point, say $a$, then $f$ has no other fixed point since $f$ is assumed to be different from the identity. Setting $z=a$ in (4.1) we conclude that $S(a)=a, S \in \Gamma$. This implies that $\Gamma$ is finite and hence everywhere properly discontinuous. Contradiction. Hence $f$ does not have a fixed point. We may therefore apply the result of Denjoy and Wolff cited in $\S 3$ to $T^{-1} \circ f \circ T$, where $T$ is a univalent conformal map of the open unit disk onto the upper half-plane. Using the functional equations induced from (4.1) we conclude that all $S \in \Gamma$ have a common fixed point on $R \cup\{\infty\}$. Indeed, if $F$ is a holomorphic map of the open unit disk into itself without a fixed point and $A$ is a Möbius transformation mapping the open unit disk onto itself and such that $F[A(z)]=A[F(z)],|z|<1$, then the $\eta$ of $\$ 3$ pertaining to $F$ is a fixed point of $A$ since $A^{-1}(\eta)$ satisfies the same condition as $\eta$.

It is routine to conclude that $\Gamma$ is cyclic. For either the $S$ other than the identity are all parabolic or else are all hyperbolic and have the same fixed points. The proof is immediate on reduction to the case where the common fixed point is $\infty$. Thus if $S_{1}(z)=z+a, a \in R-\{0\}, S_{2}(z)=\rho z, 0<\rho<1$, we have

$$
S_{2} \circ S_{1} \circ S_{2}^{-1}(z)=z+\rho a,
$$

and we conclude that $\Gamma$ is not properly discontinuous at any point since we may replace $\rho$ by $\rho^{n}$ where $n$ is a positive integer. If now $S_{1}(z)=\sigma(z-a)+a$, where $\sigma$ is positive but different from 1 , then

$$
S_{2} \circ S_{1} \circ S_{2}^{-1}(z)=\sigma z+\rho(1-\sigma) a,
$$


and we conclude that $\Gamma$ is not properly discontinuous at any point. Hence $\Gamma$ is cyclic and hence is not properly discontinuous at most at two points. The hypothesis on $\Gamma$ is violated. We conclude that under the assumption that $f$ maps the upper half-plane into itself $f$ is the identity.

There remains to be considered the case where $f$ does not map the upper half plane into itself. By virtue of the fact that $f$ does not take real values, in this case $f$ maps the upper half-plane into the lower half-plane. We extend the definition of $f$ to $\widetilde{c}-R$ by Schwarzian reflexion. We denote the so-defined extension of $f$ by $f_{1}$ and define $f_{2}=f_{1} \circ f_{1}$. Now $f_{2}$ satisfies the system (4.1), in fact for $z \in \mathbb{E}-R, S \in \Gamma$. Hence by the result of the first case we see that $f_{2}$ is the identity map. Consequently, $f_{1}$ is the restriction to its domain of an involutary elliptic Möbius transformation $g$. By normalization we may take the fixed points of $g$ to be 0 and $\infty$. When this reduction is made, we see that

$$
g[S(a)]=S[g(a)]=S(a),
$$

$a=0, \infty$. Hence $\{S(0), S(\infty)\}=\{0, \infty\}$ and $S \circ S$ has 0 and $\infty$ as fixed points. Thus if $S$ is not the identity, $S$ is hyperbolic with fixed points 0 and $\infty$ or else $S$ is elliptic and $S(z)$ is of the form $c / z$ where $c$ is a negative real number. If $S_{1}$ and $S_{2}$ are two distinct elliptic transformations of this form, $S_{1}^{-1} \circ S_{2}$ is hyperbolic and has 0 and $\infty$ as fixed points. We are led to the conclusion that $\Gamma$ fails to be properly continuous at most at 0 and $\infty$. The hypothesis on $\Gamma$ is contradicted. Hence the assumption that $f$ does not map the upper halfplane into itself is to be rejected.

The lemma is thereby established.

Remark. It is readily concluded by Schwarzian reflexion that Lemma A remains valid when the domain of $f$ is taken to be the lower half-plane.

5. We now proceed to the second lemma. We recall that a group $\Gamma$ which is not properly discontinuous at any point of $R \cup\{\infty\}$ is said to be of the first kind. This is the case for the modular group. A group $\Gamma$ which is not of the first kind is said to be of the second kind. Our second lemma holds for groups of the first kind but does not hold for all groups of the second kind having more than two points where they fail to be properly discontinuous. [In fact, examples may be constructed to show the failure of the lemma for groups of the latter type with the aid of a Schottky doubling of a plane region of finite connectivity 
greater than two having regular analytic Jordan boundary and the mapping theorem for planar surfaces.]

Lemma B. Let $f$ be meromorphic on the upper half-plane, attain a real value at some point, and satisfy the system of equations (4.1) where now $\Gamma$ is taken to be of the first kind. Then $f$ attains every point of the extended plane, in fact at infinitely many points mutually non-equivalent relative to $\Gamma$.

Proof. Use will be made of two results. The first, which is standard and concerns the proper discontinuity at a point $a$ of a group $G$ of Möbius transformations, states: $G$ is properly discontinuous at $a$ if and only if $G$ is normal at $a$ and there exists a neighborhood $V$ of $a$ such that $T(a) \in V$ for only a finite set of $T$ in $G$. The second result states that if a family of Möbius transformations is not normal at a point $a$, then for each neighborhood $V$ of $a$ there exists a univalent sequence $\left(f_{n}\right)$ of members of the family such that with at most one exception each point of the extended plane belongs to $f_{n}(V)$ for infinitely many $n$. [The latter result may be established as follows. We take $V$ as an open disk, as we may, and $\left(f_{n}\right)$ as a univalent sequence of members of the family no subsequence of which converges uniformly in $V$. We assume, as we may, that $\left(f_{n}\right)$ has been so chosen that there exists a value belonging to $f_{n}(V)$ for all $n$. We normalize so that the value in question is $\infty$. Suppose that a value $b$ belongs to $f_{n}(V)$ for only finitely many $n$. We assume, as we may, that $b$ is omitted. The set of values omitted by every $f_{n} / V, n \geq m$, is a compact convex set containing $b$. If it did not reduce to $b$, the family $\left\{f_{n} / V\right\}$ would be normal. But this is impossible. It follows that all values distinct from $b$ lie in $f_{n}(V)$ for infinitely many $n$.]

We turn to the proof of Lemma B. Let $f$ attain the real value $a$. By virtue of the first of the cited results one of two alternatives occurs.

(1) $\Gamma$ is normal at $a$ but there exists a univalent sequence of members of $\Gamma$, say $\left(T_{n}\right)$, converging uniformly in some open disk centered at $a$ and such that $\left(T_{n}(a)\right)$ tends to $a$. The limit function must be the constant taking the value $a$, for otherwise $\Gamma$ would not be properly discontinuous at each point of the upper half-plane. Now if the limit function is constant, then for $n$ large $T_{n}$ is hyperbolic and has a fixed point near $a$. This is not compatible with the assumed normality of $\Gamma$ at $a$. Hence this alternative must be rejected.

(2) $\Gamma$ is not normal at $a$. Since $\Gamma$ is of the first kind, no orbit 


$$
\{T(z): T \in \Gamma\}
$$

reduces to a point for otherwise $\Gamma$ would be cyclic and hence not of the first kind. This observation taken with the non-normality of $\Gamma$ at $a$ implies that if $V$ is a neighborhood of $a$, then each point of the extended plane belongs to $T(V)$ for infinitely many $T \in \Gamma$.

We turn to $f$ and suppose that $f(b)=a, a$ real, $\operatorname{Im} b>0$. Let $V$ be an open neighborhood of $b$ lying in the upper half-plane. Now $f[T(V)]=T[f(V)]$ and $f(V)$ is a neighborhood of $a$. We assume, as we may, that $b$ is not a fixed point of any member of $\Gamma$ other than the identity and take $V$ so small that the $T(V)$ are mutually disjoint. It follows that no two distinct points of $V$ are equivalent relative to $\Gamma$. We see that each point of the extended plane lies in $f[T(V)]$ for infinitely many $T$. We now show that given $w$, a point of the extended plane, there exists a univalent sequence $\left(z_{n}\right)$ of points in $V$ such that $w$ is attained by $f$ at some point of the orbit $\left\{T\left(z_{n}\right): T \in \Gamma\right\}, n=0,1, \ldots \ldots$. It suffices to proceed recursively. Clearly, there is a point, say $z_{0}$, in $V$ such that $w$ is attained at a point of the orbit $\left\{T\left(z_{0}\right): T \in \Gamma\right\}$. We thereupon take $V_{1}=V-\left\{z_{0}\right\}$ and note that $f$ takes a real value at some point of $V_{1}$. Hence there exists $z_{1} \in V_{1}$ such that $w$ is attained at some point of $\left\{T\left(z_{1}\right): T \in \Gamma\right\}$. The inductive construction of $\left(z_{n}\right)$ is now clear. At the $(n+1)$ st stage we have $V_{n+1}=V-\left\{z_{0}, \ldots \ldots, z_{n}\right\}$. At some point of this set $f$ takes a real value.

The lemma is established.

6. The function $F$ is now easily treated. Since $F$ restricted to the upper half-plane satisfies (2.2) and is not the identity $[F(i)=-i]$, it follows from Lemma A that $F$ attains a real value at some point of the upper half-plane. The desired result follows on application of Lemma B since the modular group is indeed of the first kind. 